\title{
Educational Interventions to Improve Atopic Dermatitis Outcomes for Children: A Systematic Review
}

\author{
Tamarachiara Kuntjoro, Adeline Jaclyn, Myrna Adiwijaya \\ Duta Indah Hospital, Jakarta, Indonesia
}

\begin{abstract}
Background: Atopic dermatitis (AD) has a significant negative impact on the quality of life (QoL) of affected children and their families. Despite the availability of efficacious treatment, poor knowledge of AD treatment, and adherence to daily controller medications leads to a high rate of treatment failure. Purpose: To characterize the efficacy of educational interventions to improve $\mathrm{AD}$ outcomes in the pediatric population. Methods: A systematic search of PubMed, OVID Medline, CINAHL, and PsychINFO was conducted from September 2010 through September 2018. We compared populations, intervention characteristics, study designs, outcomes, settings, and intervention levels across studies. Result: Of 85 articles identified and reviewed, 7 articles met inclusion criteria. All included studies demonstrated efficacy in overall outcomes. All studies were performed in a hospital setting or utilized technology. The most common format for pediatric $\mathrm{AD}$ intervention delivery was the use of group lectures. Promising strategies to address time limitations in parents of children with $\mathrm{AD}$ include multifaceted educational support programs, individually tailored nurse consultation, and WebBased Educational Programs. This systematic review is not a meta-analysis, therefore limiting its quantitative assessment of studies. Conclusion: Educational interventions demonstrate efficacy in improving pediatric AD outcomes. As the seven included studies employed a wide variety of outcome assessment tools, it is difficult to compare the extent of improvement in outcomes across these studies. In order to better assess the comparative efficacy of different educational interventions to improve outcomes in children with $\mathrm{AD}$, it is essential to standardize outcome measures.
\end{abstract}

Key words: atopic dermatitis, children, educational intervention, efficacy, outcome.

Correspondence address: Tamarachiara Kuntjoro, Duta Indah Hospital, Jl. Teluk Gong Raya 12, Jakarta, Indonesia. Phone: (+62) 81223994591, email: tamarachiar@gmail.com.

\section{INTRODUCTION}

Atopic dermatitis (AD) is the most common cutaneous disease in the pediatric population, ${ }^{1,2}$ currently affects up to $15-20 \%$ of children worldwide, ${ }^{1-}$ ${ }^{4}$, and its prevalence continues to rise. ${ }^{1,3}$ This waxing and waning inflammatory skin disorder has a debilitating impact on the quality of life (QoL) of affected children. ${ }^{3}$ Furthermore, parents report emotional stress in dealing with their child's AD. ${ }^{3,4}$ It declines family functioning, and in turn, results in decreased child well-being and social functioning. ${ }^{3}$ Studies demonstrate that $\mathrm{AD}$ patients are frequently inadequately treated. ${ }^{4}$ The majority of therapeutic failures are attributed to poor adherence to prescribed treatment. ${ }^{1}$ Effective management of $\mathrm{AD}$ requires knowledge about $\mathrm{AD}$ and medications to treat this condition, and self-management skills to achieve disease control. ${ }^{2}$ Educational interventions have demonstrated efficacy in improving treatment adherence and QoL for patients and their caregivers in AD management. Educational self-management interventions empower patients to manage their disease by combining skills acquisition and information dissemination techniques. ${ }^{6}$ In pediatric patients with $\mathrm{AD}$, educational interventions primarily target the parents. ${ }^{5}$ Core educational concepts include: the understanding of no cure and remitting-relapsing course of the disease; the ability to control, rather than cure the disease, allows for improved QoL; the skills on how to effectively apply topical therapy; and the importance of continuation of therapy despite remission. ${ }^{1,3}$ Currently, educational interventions to promote $\mathrm{AD}$ management vary across settings; educational topics covered; number, duration of and frequency of sessions; group and individual formats; and agent of delivery employed. ${ }^{5,7}$ The goal of this systematic review was to identify and evaluate recently published educational interventions to improve $\mathrm{AD}$ outcomes in the pediatric population. Interventions demonstrating efficacy may be used in clinical practice as well as be integrated into future multilevel effectiveness studies.

\section{METHODS}

We completed a systematic review of the literature in the spirit of the Preferred Reporting Items for Systematic Reviews and Meta-Analysis (PRISMA) reference standards. ${ }^{8}$ The search was completed using the following databases: PudMed Medline, OVID Medline, CINAHL, and PsychINFO (Table 1). This 
search of titles and abstracts was limited to articles focusing on humans and published in the English language between September 2010 and September 2018. To identify educational and self-management intervention studies for pediatric patients with $\mathrm{AD}$, we combined the terms atopic dermatitis and intervention with either educational, self-management technology or children. In addition, we reviewed the references of included studies for additional studies of interest.

For article selection, we included intervention studies of educational and self-management interventions for $\mathrm{AD}$ in children. Articles were excluded if they were not full-manuscripts, not AD focused, not focused on the pediatric age group, or not educational and/or self-management interventions (Figure 1).

Table 1. PubMed search strategy

(Atopic dermatitis [Title/Abstract]) OR (Atopic eczema [Title/Abstract]) AND (Intervention

[Title/Abstract]) AND (Education [Title/Abstract]) OR (Technology [Title/Abstract]) OR (Selfmanagement [Title/Abstract]) AND (Child [Title/Abstract]) or (Pediatric [Title/Abstract])

Limited to literature published from September 2010 through September 2018, English Language and Humans
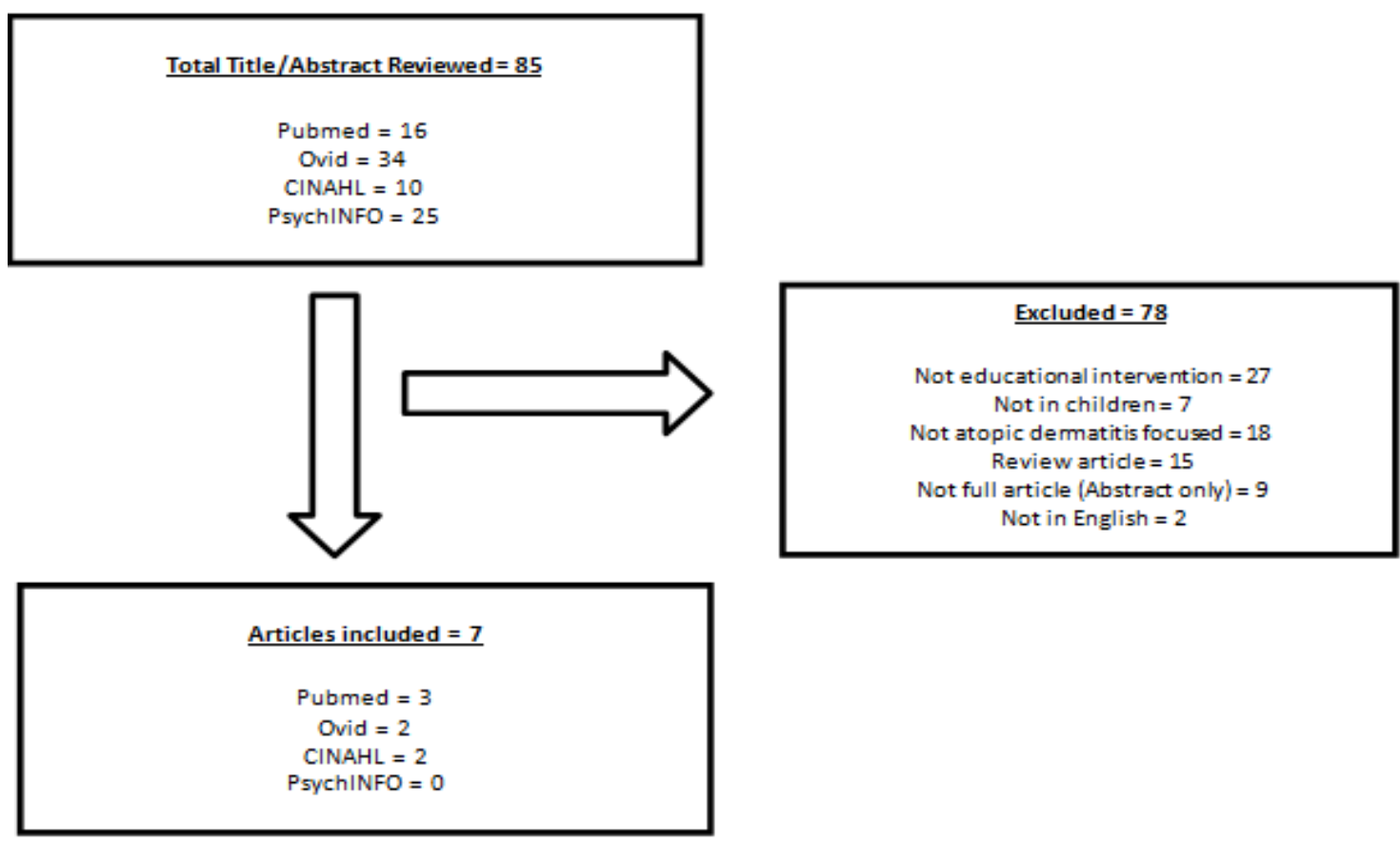

Figure 1. Consort diagram. The diagram illustrates the inclusion and exclusion criteria for the articles included in this systematic review.

Seven articles met our inclusion and exclusion criteria, as described in Table 1 for grouping of intervention studies. For each article, the sample size, population, intervention, control, outcome/result, timeframe, setting, and intervention level were summarized. Intervention level was determined to be either single or multilevel; levels included children with $\mathrm{AD}$, his/her parents or caregivers, and community. If specified, outcomes were reported as primary and secondary. All outcomes and results were listed as positive $(p \leq 0.05)$ or negative $(p \geq 0.05)$.

\section{RESULTS}

Of the seven studies included, five were single level, targeting parents or caregivers of children with AD. ${ }^{9,10,12,14,15}$. The other two were multilevel and

targeted mother-child dyads of children with $\mathrm{AD} .^{11,13}$ None of the studies included a community-level intervention.

All of the five interventions addressing parents or caregivers only reported positive outcomes. ${ }^{9,10,12,14,15}$ However, one intervention had negative outcomes on topical corticosteroid use and anxiety level but yielded positive effects on other outcome measurements. ${ }^{10}$ Four out of the single-level studies were conducted in Europe, but targeted a variety of age groups: Mason et al. ${ }^{9}$ (range 3 months- 6 years), Rolinck-Werninghaus et al. $^{14}$ (mean age $=1.7$ years, $25 / 75^{\text {th }}$ Percentile 0.7/4.0), Pustisek et al. ${ }^{10}$ and Breuer et al. ${ }^{12}$ (range 3 months-7 years). Gilliam et al. focused on children aged 1 month-12 years in the United States. ${ }^{15}$ Studies featured a range of key intervention components to 
facilitate the clinic verbal instructions, including the production and dissemination of $\mathrm{AD}$ educational videos; ${ }^{9} \mathrm{AD}$ educational booklets; ${ }^{10}$ Eczema Action Plans (EAP); ${ }^{15}$ symptom monitoring diaries; 9,10 AD interdisciplinary lectures, ${ }^{10,12}$ individually tailored AD consultations; ${ }^{14}$ and AD telephone helpline. ${ }^{9}$ Most studies $(\mathrm{n}=3)$ included one component, ${ }^{12,14,15}$ whereas 2 studies featured multiple components. ${ }^{9,10}$ Both Pustisek et al. and Breuer et al. utilized 2-hour lectures delivered in group sessions. ${ }^{10,12}$ Interventions were delivered across two settings: hospital, ${ }^{10,12,14,15}$ and online. ${ }^{9,14}$ Duration of follow-up varied greatly across studies, ranging from 14 days to 12 months. ${ }^{9,10,12,14,15}$

Positive outcomes included use of topical emollient ${ }^{9}$ severity of AD by means of PatientOriented Eczema Measure (POEM) score; ${ }^{9}$ Patient Eczema Severity Time (PEST) score ${ }^{9}$ PatientOriented Scoring Atopic Dermatitis (PO SCORAD) index; ${ }^{10}$ Scoring Atopic Dermatitis (SCORAD) index; ${ }^{10,12}$ sleep disturbance; ${ }^{9,14}$ number of general practitioner (GP) visits, ${ }^{9}$ parental feelings of control $;{ }^{9}$ steroid prescriptions; ${ }^{9}$ cost of care ${ }^{9}$ topical corticosteroid use; ${ }^{12}$ childhood eczema study questionnaire; ${ }^{15}$ parents' quality of life through Fragebogen für Eltern von Kindern mit Neurodermitis (FEN); ${ }^{12}$ AD knowledge; ${ }^{12}$ parents' self-confidence; ${ }^{14}$ and pruritus. ${ }^{14}$ Two studies utilized control groups of standard care only, ${ }^{10,12,15}$ and the remaining studies did not employ control groups. ${ }^{9,14}$ A study with mixed outcomes was the one conducted by Gilliam et al., evaluating the use of the EAP versus verbal instruction alone during standard care. Gilliam et al. performed a randomized, controlled pilot study on 88 children with $\mathrm{AD}$ and utilized the childhood eczema study questionnaire to measure intervention outcomes. The questionnaire was comprised of 20 items with four major domains, namely QoL, understanding of skincare, symptoms of the disease, and family satisfaction. The study demonstrated improvement in the overall score of the outcomes significantly $(\mathrm{p}=$ 0.019). The QoL and symptoms of disease domains showed significant improvement with respective $\mathrm{p}=$ 0.004 and $p=0.04$, but the understanding of skincare and family satisfaction domains did not show any improvement. ${ }^{15}$ For further details on this questionnaire refer to the accompanying Table 2 .

The two interventions targeting both children with $\mathrm{AD}$ and their mothers showed improvement in outcomes. $^{11,13}$ However, one of the studies reported a negative outcome on the amount of corticosteroid use and described a non-significant improvement in the parental quality of life. ${ }^{11}$ Futamura et al. delivered a 2day parental education program (PEP), including practical skills training to 59 children with $\mathrm{AD}$ (range
$=6$ months- 6 years) and their mothers simultaneously. This study utilized a randomized controlled trial design and had a 6-month follow-up period.

It was conducted in Japan and delivered in a hospital setting. ${ }^{11}$ Son et al. directed the educational interventions on 40 children with $\mathrm{AD}$ aged under 3 years and their mothers. This study leveraged technology for intervention delivery featuring webbased tailored $\mathrm{AD}$ education sessions, knowledge assessment (via online quiz), and practice assessment (via online self-checklist). The outcomes were selfreported and assessed electronically utilizing questionnaires via e-mail or SMS. This study was executed in Korea and had a short 2-week period of follow-up. ${ }^{13}$ Positive outcomes of these two studies were SCORAD index $;{ }^{11}$ objective SCORAD index; ${ }^{11}$ sleeplessness symptom score; ${ }^{11}$ corticosteroid anxiety score; ${ }^{11}$ disease severity; ${ }^{13}$ QoL of children; ${ }^{13}$ and mothers' self-efficacy. ${ }^{13}$ Both multilevel studies employed a control group. ${ }^{11,13}$ One utilized a usual care group, ${ }^{13}$ and the other employed a usual care group with a booklet about $\mathrm{AD} .^{11}$

\section{DISCUSSION}

Patient education for AD demonstrates improved treatment adherence, QoL, and self-management skills. ${ }^{3,14}$ This systematic review sought to assess educational intervention studies in pediatric $\mathrm{AD}$ to verify efficacious approaches for potential integration into clinical practice and inclusion in future multilevel intervention studies. All of the seven studies were performed in a hospital setting or by leveraging technology. ${ }^{9-15}$ No studies targeted community-level interventions. Two of the seven studies did not include a control group. ${ }^{9,14}$ In general, studies demonstrated improved outcomes. ${ }^{9-15}$ Our systematic review has several limitations, including publication bias. Although we did not execute a meta-analysis, we carefully followed the spirit of the Preferred Reporting Recommendations for Systematic Reviews and Metaanalysis. ${ }^{8}$

In summary, this evidence demonstrates the efficacy of a variety of educational interventions to improve outcomes in children with AD. Group lectures were the most common form of intervention delivery. ${ }^{10-12}$ These interventions merit further study in order to evaluate the optimal duration and frequency of group lectures. Multifaceted educational support programs, ${ }^{9}$ single individually tailored nurse consultation, ${ }^{14}$ and web-based education program (WBEP) were promising educational approaches that allow greater scheduling flexibility. ${ }^{13}$ Finally, future research on educational interventions in pediatric $\mathrm{AD}$ would benefit from the use of standardized outcome 
measures to allow better comparison of outcomes across studies.

\section{REFERENCES}

1. Kotrulja L, Milavic T, Bulic SO, Situm N, Konsuo AB, Mursic I, et al. Importance of educational intervention and parental knowledge on atopic dermatitis in children. Acta Clin Croat 2016;55:29-34.

2. LeBovidge JS, Elverson W, Timmons $\mathrm{KG}$, Hawryluk EB, Rea C, Lee $M$, et al. Multidisciplinary interventions in the management of atopic dermatitis. J Allergy Clin Immunol 2016;138(2):325-34.

3. Lifschitz $C$. The impact of atopic dermatitis on quality of life. Ann Nutr Metab 2015;66(1):34-40.

4. Heratizadeh A. Therapeutic Patient Education. Curr Treat Options Allergy 2014;1:358-64.

5. Ersser SJ, Cowdell F, Latter S, Gardiner E, Flohr C, Thompson AR, et al. Psychological and educational interventions for atopic eczema in children (Review). Cochrane Database Syst Rev 2014;1:1-57.

6. Stalder JF, Bernier C, Ball A, Raeve LD, Gieler U, Deleuran M, et al. Therapeutic patient education in atopic dermatitis: worldwide experiences. Pediatr Dermatol 2013;30(3):329-34.

7. Sidbury R, Tom WL, Bergman JN, Cooper KD, Silverman RA, Berger TG, et al. Guidelines of care for the management of atopic dermatitis. J Am Acad Dermatol 2014;70:338-51.

8. Liberati A, Altman DG, Tetzlaff J, Mulrow C, Gotzsche P, Loannidis JPA, et al. The PRISMA statement for reporting systematic reviews and meta-analyses of studies that evaluate health care interventions: explanation and elaboration. PLoS Med 2009;6(7):1-28.
9. Mason JM, Carr J, Buckley C, Hewitt S, Berry P, Taylor $\mathrm{J}$, et al. Improved emollient use reduces atopic eczema symptoms and is cost neutral in infants: before-and-after evaluation of a multifaceted educational support program. BMC Dermatol 2013;13:7.

10. Pustisek N, Situm M, Zivkovic MV, Hadzavdic SL, Vumek M, Niseteo T. The significance of structured parental educational intervention on childhood atopic dermatitis: a randomized controlled trial. JEADV 2016;30:806-12.

11. Futamura M, Masuko I, Hayashi K, Ohya Y, Ito K. Effect of a short-term parental education program on childhood atopic dermatitis: a randomized controlled trial. Pediatr Dermatol 2013;30(4):43843.

12. Breuer K, Matterne U, Diepgen TL, Fartasch M, Gieler U, Kupfer J, et al. Predictors of benefit from an atopic dermatitis education program. Pediatr Allergy and Immunol 2014;25:489-95.

13. Son HK, Lim Jiyoung. The effect of a web-based education program (WBEP) on disease severity, quality of life, and mother's self-efficacy in children with atopic dermatitis. J Adv Nurs 2014;70(10):2326-38.

14. Rolinck-Werninghaus C, Trentmann M, Reich A, Lehmann C, Staab D. Improved management of childhood atopic dermatitis after individually tailored nurse consultations: A pilot study. Pediatr Allergy and Immunol 2015;26:805-10.

15. Gilliam AE, Madden N, Sendowski M, Mioduszewski M, Duderstadt KG. Use of Eczema Action Plans (EAPs) to improve parental understanding of treatment regimens in pediatric atopic dermatitis (AD): A randomized controlled trial. J Am Acad Dermatol 2016;74(2):375-7. 


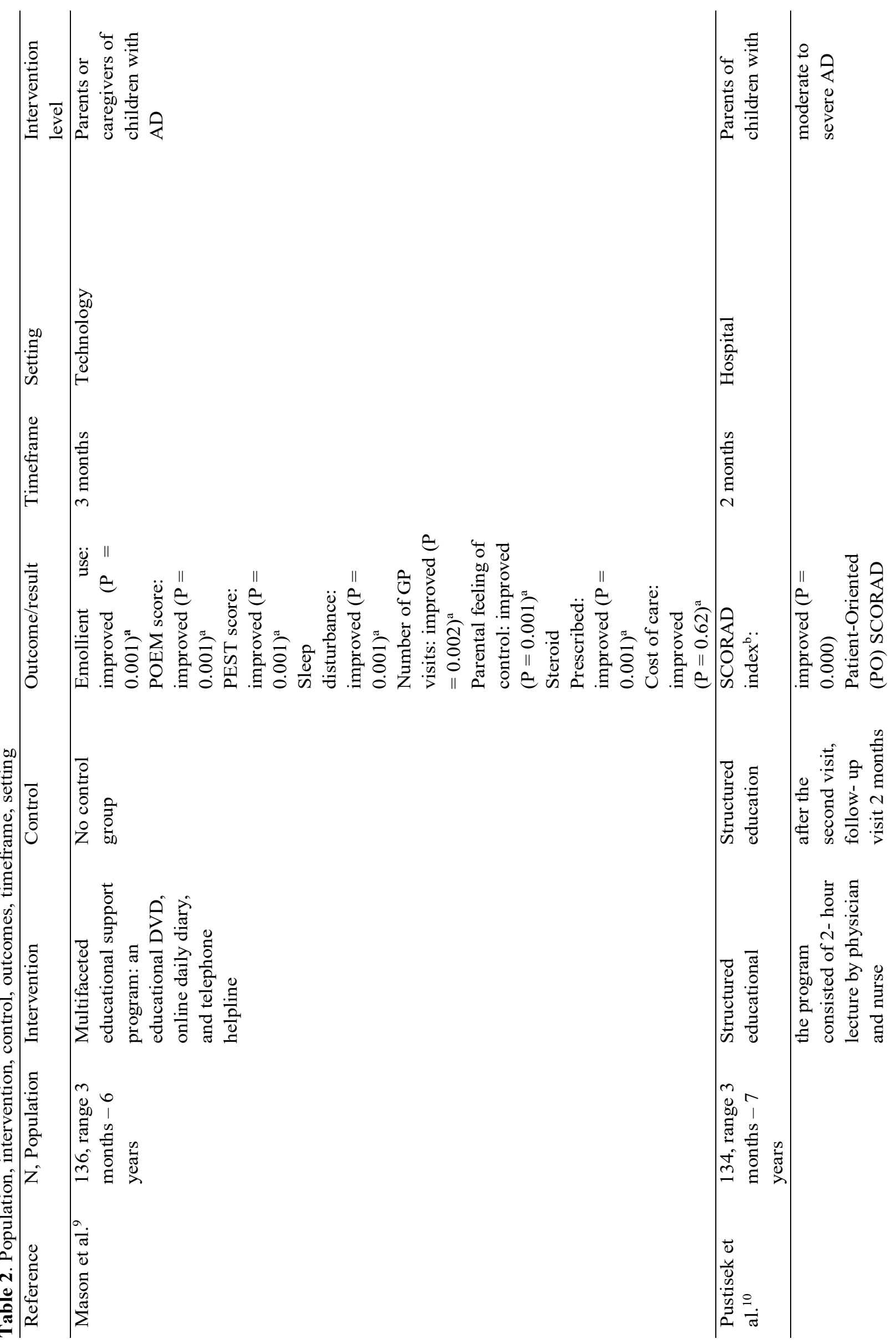




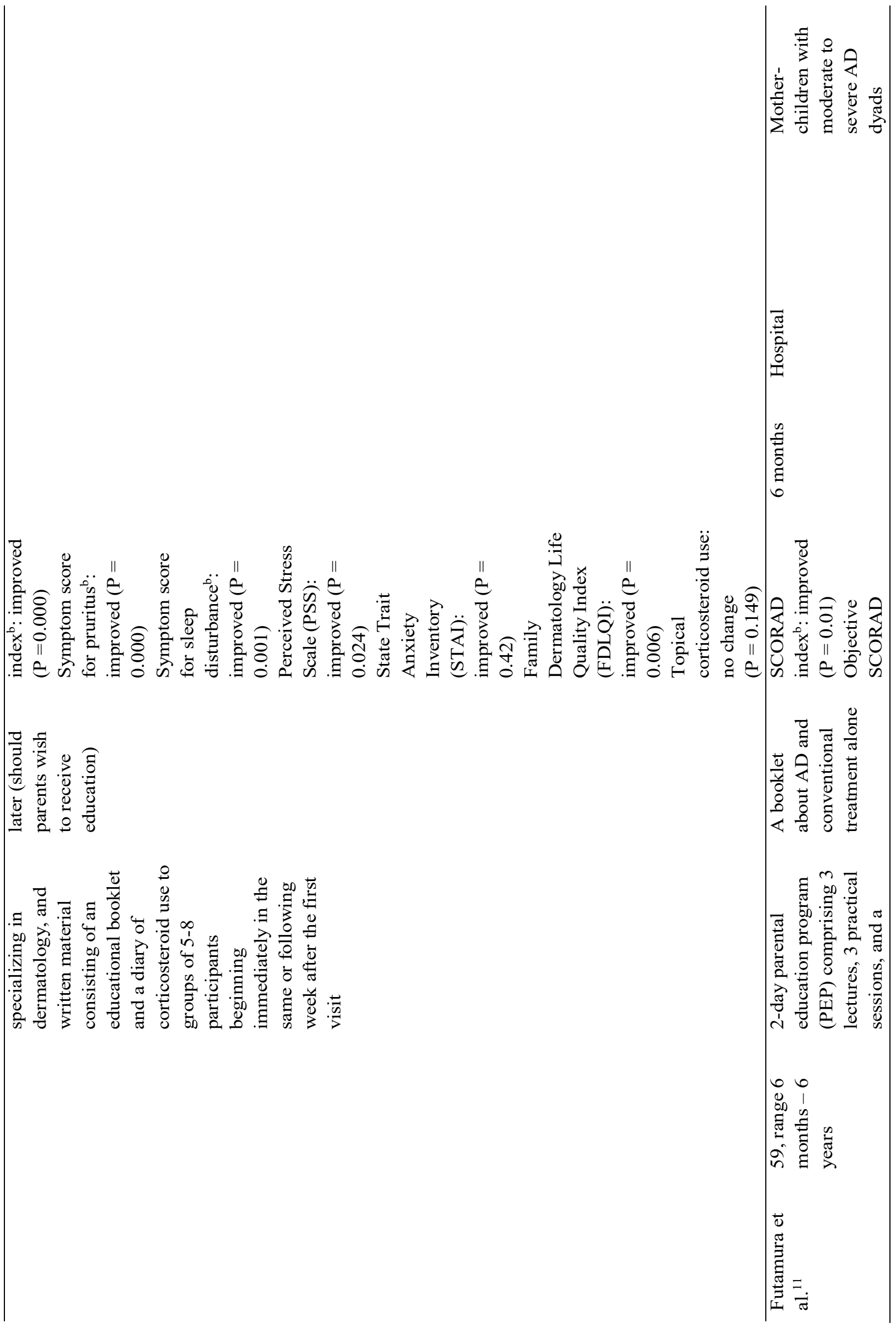




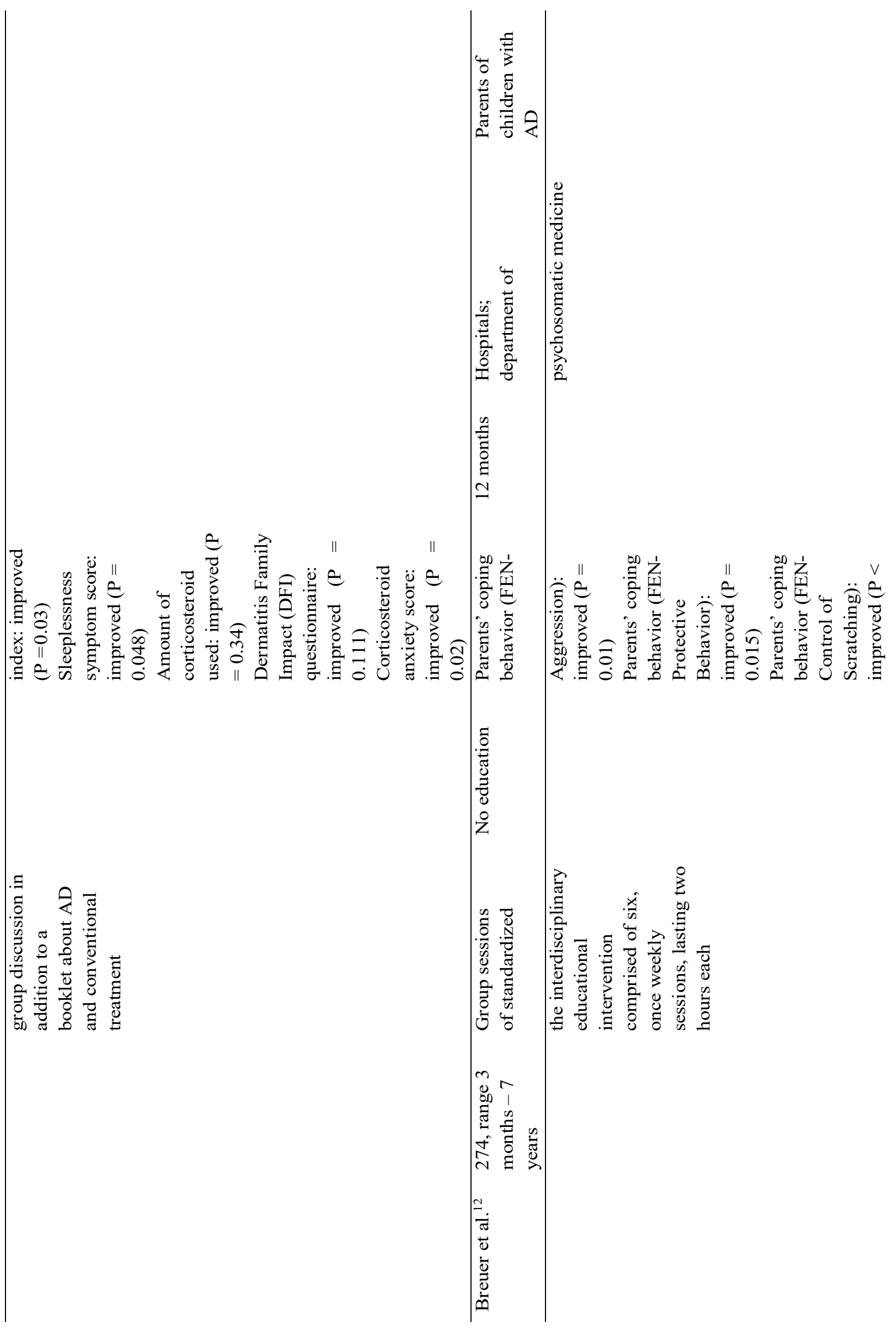




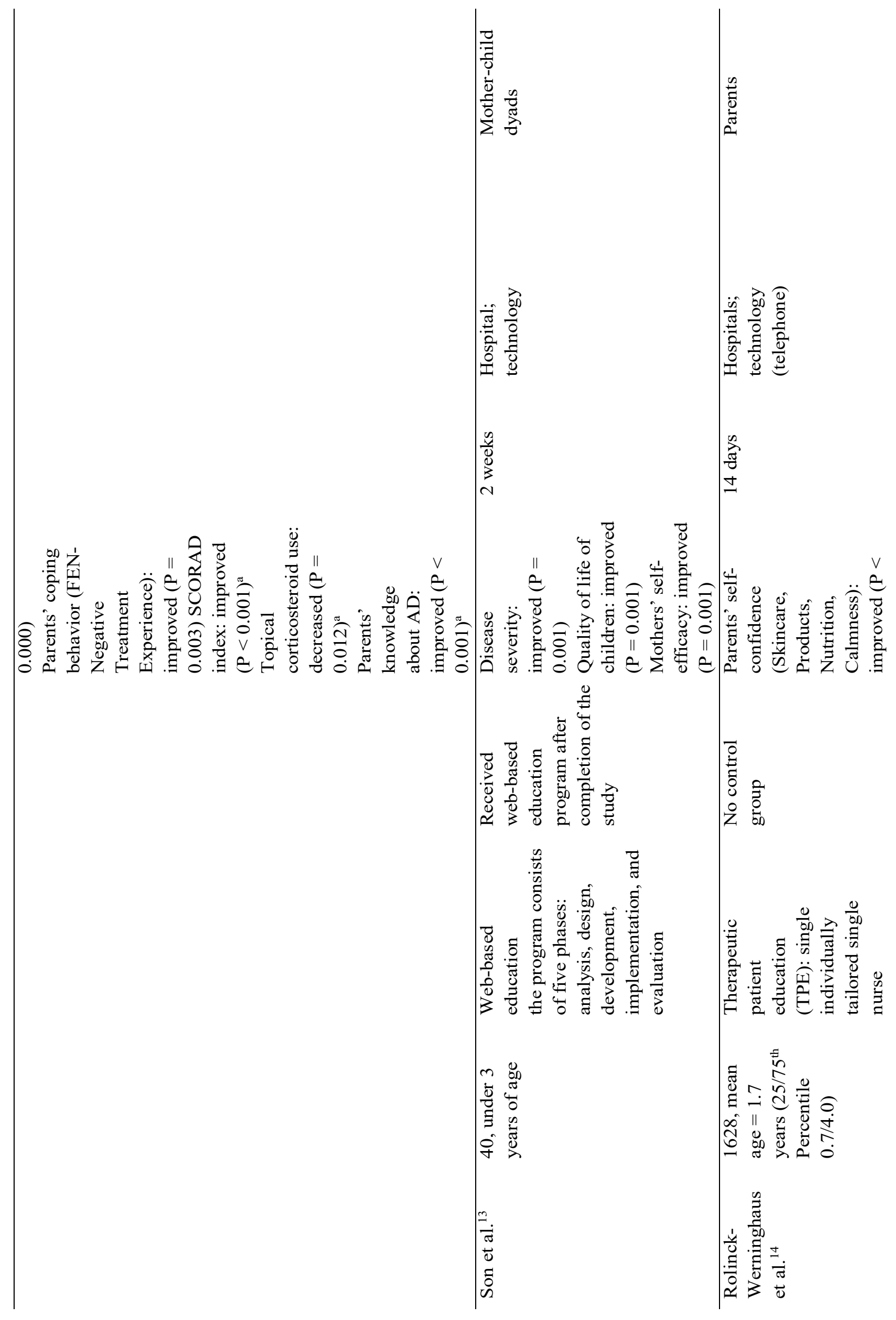




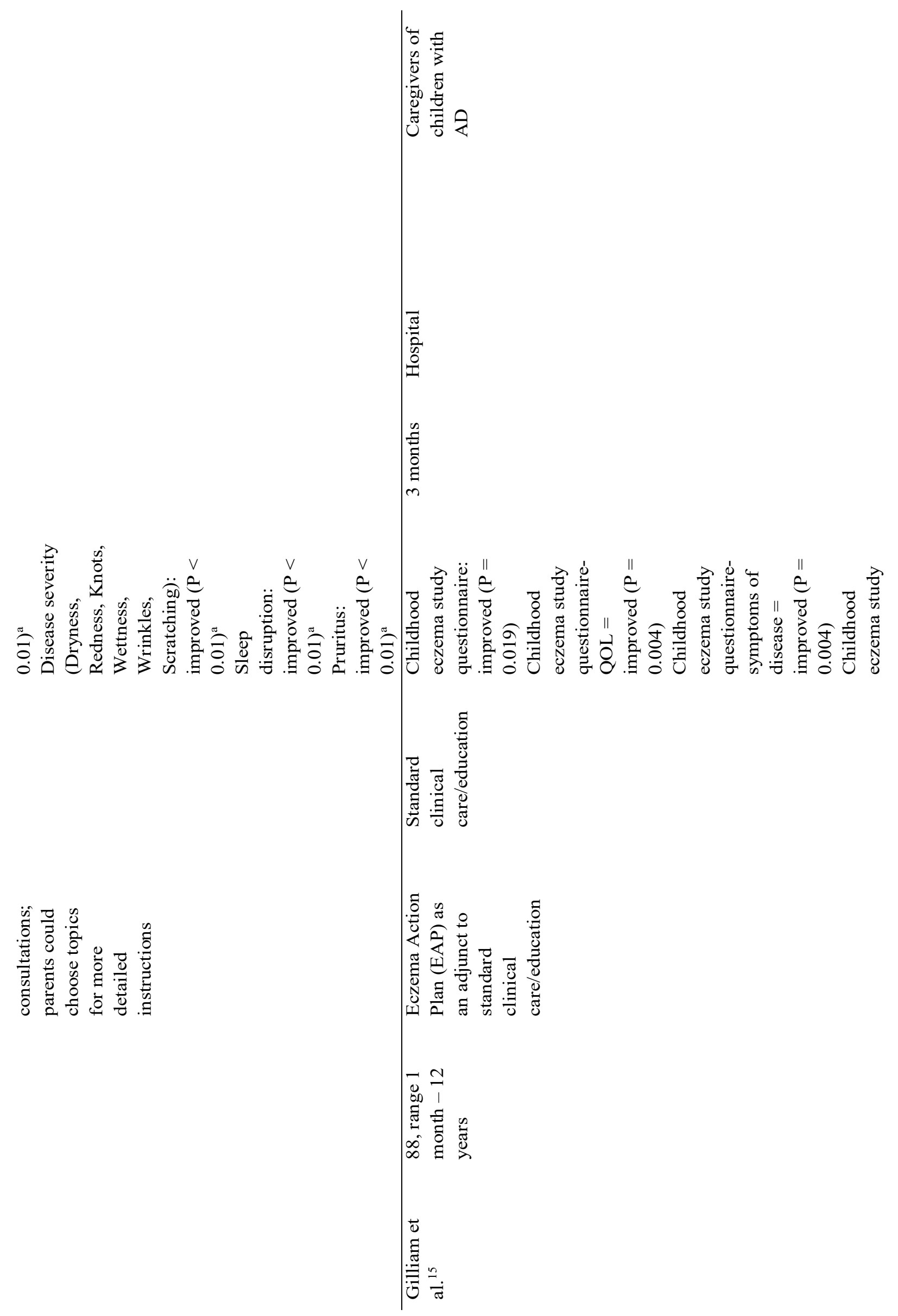




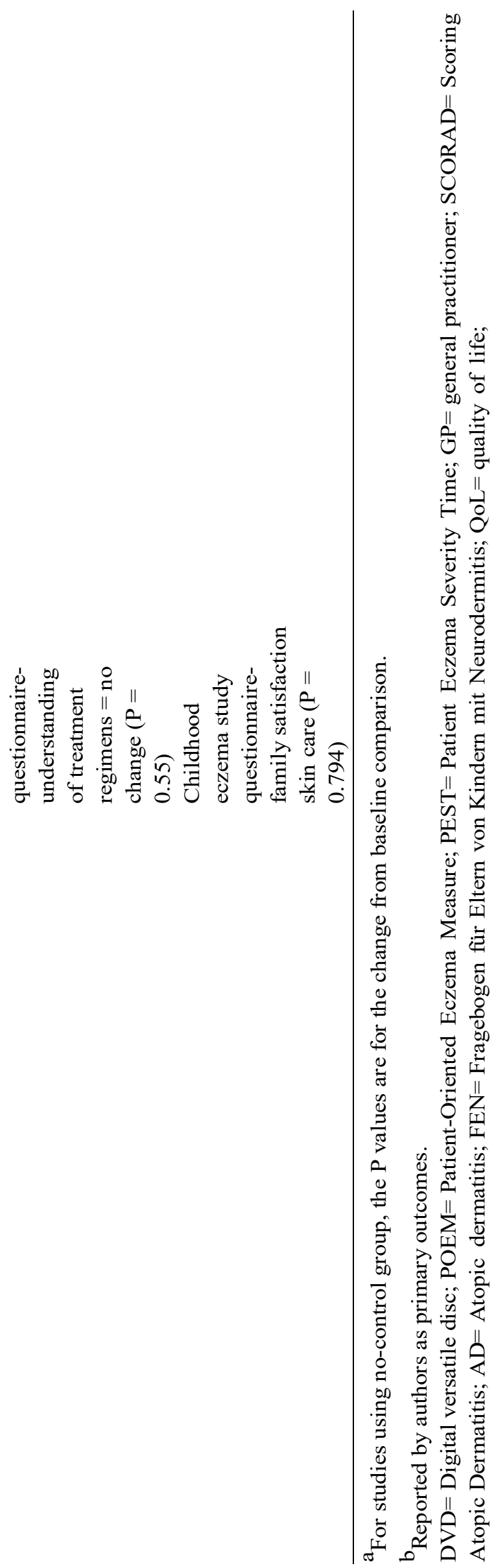

